PHYSICAL REVIEW D 92, 069902(E) (2015)

\title{
Publisher's Note: Note on bunching of field lines in black hole magnetospheres \\ [Phys. Rev. D 92, 044053 (2015)]
}

Samuel E. Gralla, Alexandru Lupsasca, and Maria J. Rodriguez

(Received 14 September 2015; published 22 September 2015)

DOI: 10.1103/PhysRevD.92.069902

PACS numbers: 04.70.-s, 04.20.-q, 11.15.Bt, 04.40.Nr, 99.10.Fg

This paper was published online on 28 August 2015 without the author's corrections incorporated into the published article. The paper has been corrected as of 3 September 2015. The text is correct in the printed version of the journal. 